\title{
Of the need to reconcile discrepancies between two different reference equations for combined single-breath DLNO-DLCO in systemic sclerosis
}

\section{To the Editor:}

Systemic sclerosis (SSc) is a chronic disease of the connective tissue with an estimated prevalence of 5.8 per 100000 in a multiethnic population living in Europe [1]. The disease mechanisms are highly complex, affecting multiple organs, including the pulmonary system. Severe pulmonary complications include SSc-associated interstitial lung disease (ILD) and pulmonary hypertension (PH), which are also associated with premature mortality [2, 3]. Early diagnosis and monitoring of pulmonary complications by spirometry, body plethysmography and pulmonary diffusing capacity are critical to lung health in SSc patients $[4,5]$. Diffusing capacity of the lung for carbon monoxide (DLCO) is a well-established and frequently applied test in SSc, and is used as a surrogate of SSc-ILD and SSc-PH progression [4, 6]. Recently, diffusing capacity of the lung for nitric oxide (DLNO) has received growing attention in the diagnostic setup of SSc patients [7, 8]; yet, its clinical value with respect to disease management and prognosis has yet to be shown. In this respect, accurate interpretation of test results using robust reference equations [5] is imperative to both clinicians and researchers. While DLNO is a promising method, uncertainty in the accuracy of available references equations limit its clinical implementation on a larger scale.

The measurement of DLNO has recently been standardised by a European Respiratory Society (ERS) Task Force [9]. The Task Force provided the largest dataset ( $n=490$, age range 18-93 years) of normal values for combined single-breath DLNO-DLCO tests for white, European and Northern American adults by pooling datasets from previously published studies [10-12]. Despite some technical and methodological inconsistencies between the different underlying studies [10-12], these reference equations are an important step towards the interpretation of DLNO tests in clinical research and practice. Recently, new reference equations for the combined single-breath DLNO-DLCO were published for European adults [13], using ERS technical standard methodology [9] and rigorous quality control in a relatively large population $(n=282)$ covering a broad age range of 18-97 years.

To date, the impact of different reference equations on the interpretation of combined single-breath DLNO-DLCO values in patient populations with pulmonary involvement is unknown. We therefore sought to compare the two most recently published reference equations for DLNO-DLCO $[9,13]$ using data from a large cohort of SSc patients with a broad range of disease severity. Those two equations were chosen as the ZAVORSKY et al. [9] equation is the official reference equation of the ERS Task Force and the MunKHOLM et al. [13] equation is the first equation that was built upon DLNO measurements following ERS Task Force standard methodology [9].

We retrospectively analysed data from an unselected cohort of clinically stable SSc patients assessed at Hôpital Cochin, Université Paris Descartes, Paris, France, between February 2007 and October 2016. 337 patients ( $84 \%$ females) with a mean \pm SD age of $54.4 \pm 13.6$ years, a body mass index of $23.7 \pm 4.4 \mathrm{~kg} \cdot \mathrm{m}^{-2}$ a forced expiratory volume in $1 \mathrm{~s}$ of $86 \pm 18 \%$ pred and a forced vital capacity of $85 \pm 18 \%$ pred were included.

@ERSpublications

This study confirms clinically relevant differences between reference equations for nitric oxide pulmonary diffusing capacity (DLNO) in systemic sclerosis. Future work on DLNO reference equations is required to improve the validity of DLNO outcomes. http://ow.ly/NnA030nxlvG

Cite this article as: Radtke T, Hua-Huy T, Dressel H, et al. Of the need to reconcile discrepancies between two different reference equations for combined single-breath DLNO-DLCO in systemic sclerosis. Eur Respir J 2019; 53: 1802109 [https://doi.org/10.1183/13993003.02109-2018]. 
Of those, 133 (39\%) and 19 (6\%) had a diagnosis of ILD and PH, respectively, and 14 (4\%) had both ILD and $\mathrm{PH}$. Combined single-breath DLNO-DLCO tests were performed using a commercially available device (HypAir; Medisoft, Dinant, Belgium) using a breath-hold time of $6 \mathrm{~s}$. The mean of two technically satisfactory tests fulfilling intertest reliability criteria [9] was used for statistical analysis. To compare DLNO and DLCO values, we calculated z-scores using the ZAvorsky et al. [9] and Munkholm et al. [13] equations.

The study complied with our institutional rules and the World Medical Association Declaration of Helsinki that deemed the study to be observational and therefore waived the need for informed consent. All functional tests were routinely performed for diagnostic and therapeutic purposes.

Our analysis revealed systematic differences (i.e. a proportional error) in DLNO and DLCO z-scores between the two equations [9, 13] (figure 1). Moreover, comparing both equations [9, 13], we noticed differential agreement of DLNO and DLCO Z-scores between sexes, with much wider limits of agreement for females (figure $1 \mathrm{~b}$ and $\mathrm{d}$ ).

The magnitudes of the difference in DLNO and DLCO z-scores between the two reference equations $[9,13]$ are likely to be multifactorial, including disparities at the population level as well as technical and
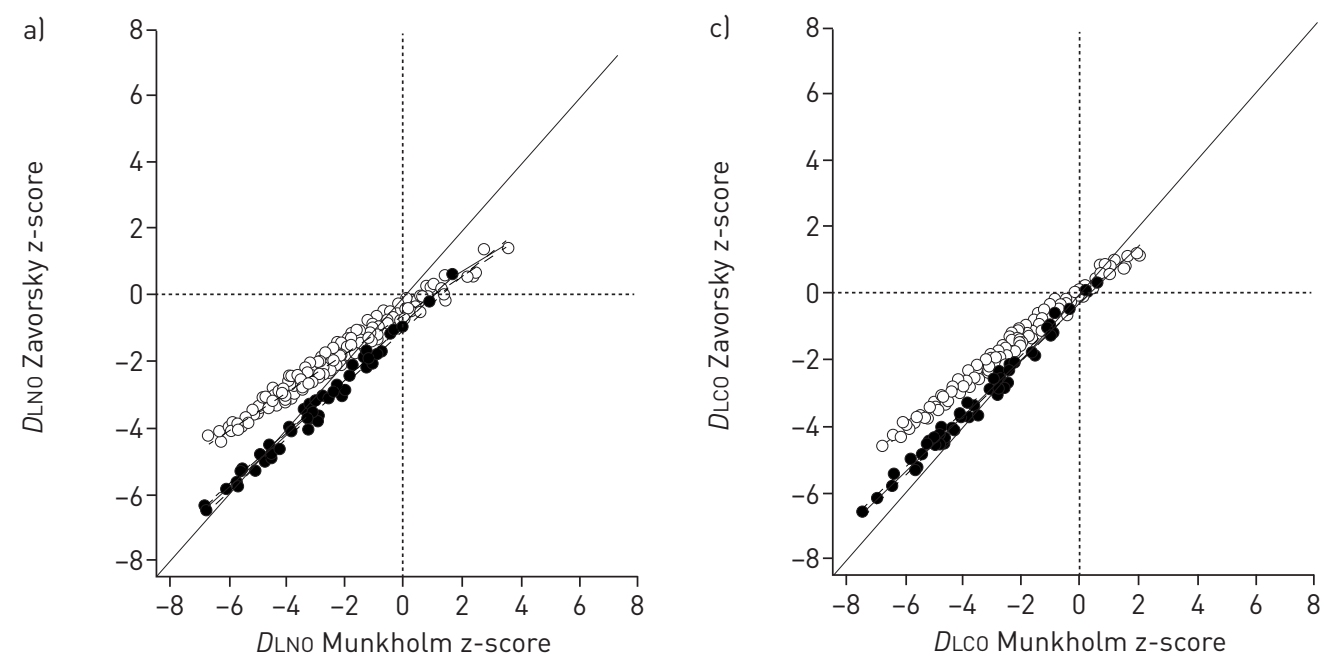

b)

Total study population $\mathrm{n}=337$ Mean bias $0.22(95 \% \mathrm{Cl} 0.14-0.30)$ Upper limit 1.72 (95\% Cl 1.58-1.86) Lower limit $-1.28(95 \% \mathrm{Cl}-1.42--1.154)$

d)
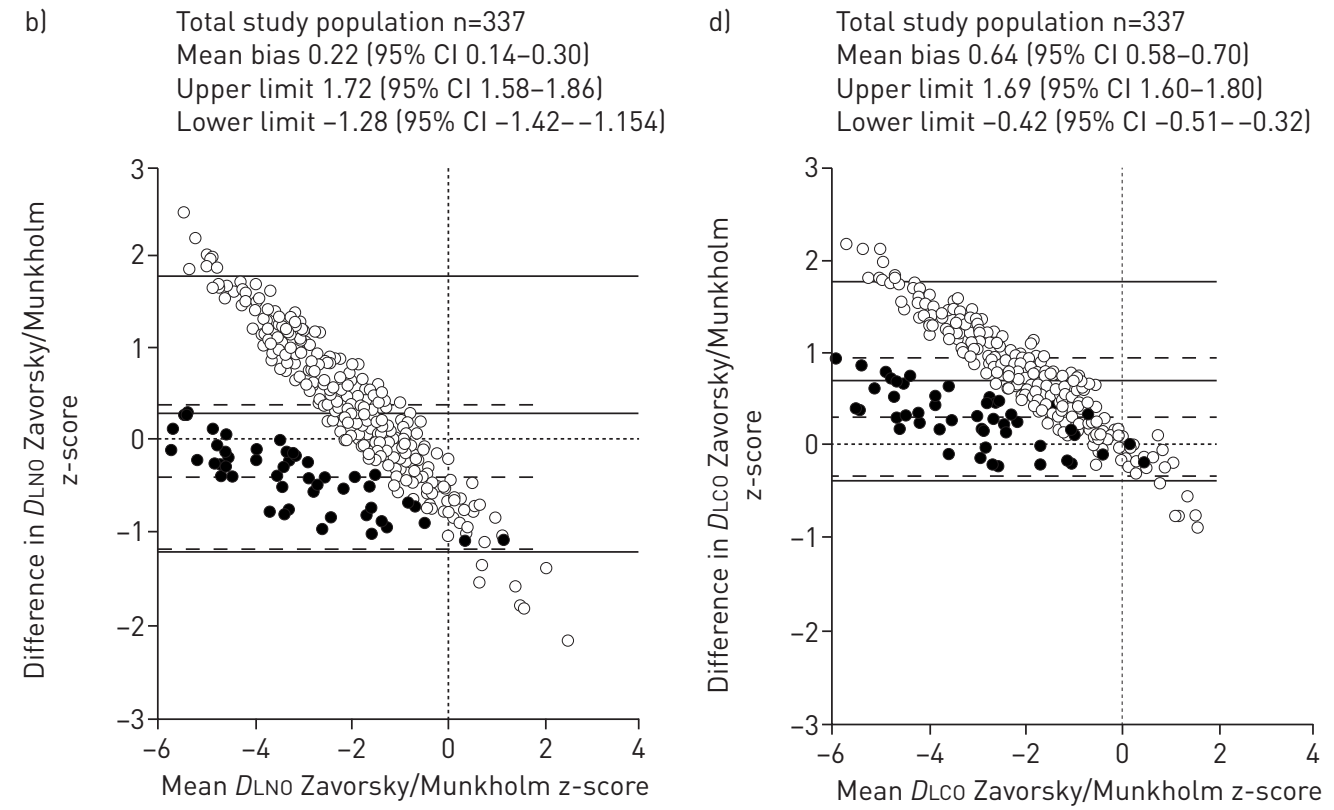

FIGURE 1 Scatter and Bland-Altman plots for comparisons between z-scores for a and b) diffusing capacity of the lung for nitric oxide $(D\llcorner N O) c$ and $d$ ) and carbon monoxide ( $D\llcorner C O)$ between reference equations published by ZAVORSKY et al. [9] and MUNKholm et al. [13]. Solid black circles represent males and open white circles represent females. The solid lines (females) and dashed lines (males) in b and d represent the mean bias and upper and lower limits of agreement (mean $\pm 1.96 \mathrm{sD}$ ). 
methodological discrepancies. However, both DLNO and DLCO were consistently different across the entire spectrum of z-scores, which points toward a systematic methodological difference. We were not able to compute z-scores for the reference equation by AgUiLANiU et al. [12], because the authors did not report the residual standard deviation for their equation. Consequently, we could not compare z-scores from this equation with those from Munkholm et al. [13] to assess the relative contribution of this study in the reference equation from ZAVORSKY et al. [9]. Nevertheless, per cent predicted values for DLNO and DLCO were, on average, $30 \%$ and $24 \%$ lower comparing Agullaniu et al. [12] with Munkholm et al. [13] equations (data not shown), and this study [12] contributed about half (54\%) of the measurements to the pooled dataset from ZaVORSKY et al. [9]. This suggests that the differences are not primarily due to differences in equipment (e.g. different devices and nitric oxide analysers) and/or breath-hold times but partially due to inclusion of data from Aguilaniu et al. [12]. For their analysis, Aguilaniu et al. [12] chose the DLNO value from the highest DLCO test and not a mean value as the other authors did $[10,11,13]$. This apparently resulted in reference equations with the highest DLNO predicted values [13]. However, to what extent the differences in per cent predicted values between the two equations impact on $\mathrm{Z}$-scores cannot be determined.

The use of different analytical approaches is another reason for the discrepancies between the two reference equations $[9,13]$. MunKHOLM et al. [13] reported sex-specific equations with residual standard errors (RSEs) separately for males and females, whereas ZAVORSKY et al. [9] did not apply sex-specific RSEs in their equation. For example, Munkholm et al. [13] uses an RSE for DLNO of 11.4 for females and 16.6 for males, while ZAVORSKY et al. [9] uses an RSE of 20.0 for both sexes. The smaller difference in standard errors between males results in different regression slopes between sexes (figure 1a and b) with an overall better agreement between DLNO Z-scores for male patients $[9,13]$. The same observation applies for DLCO. Since sex is an independent explanatory variable of DLNO $[9,13]$, and a significant and independent predictor of DLCO [14], sex-specific reference equations for combined DLNO-DLCO [10,13] are appropriate.

In conclusion, our data confirm considerable differences in DLNO and DLCO Z-scores between different references equations $[9,13]$ in a large cohort of SSc patients. In an attempt to improve the validity of reference equations, pooling of available datasets $[9,13]$ would significantly strengthen the robustness of DLNO and DLCO equations, and facilitate interpretation of pulmonary diffusing capacity measurements for both clinicians and researchers. Future work on DLNO-DLCO reference values is required to allow for calculation of sex-specific, precise lower and upper limits of normal based on a large population including people of different ethnic backgrounds, a prerequisite to evaluate ultimately and exploit the clinical potential of this promising technique.

Thomas Radtke ${ }^{1}$, Thông Hua-Huy ${ }^{2}$, Holger Dressel ${ }^{1}$ and Anh Tuan Dinh-Xuan ${ }^{2}$

${ }^{1}$ Division of Occupational and Environmental Medicine, Epidemiology, Biostatistics and Prevention Institute, University of Zurich and University Hospital Zurich, Zurich, Switzerland. ${ }^{2}$ Dept of Physiology, Cochin Hospital, Paris, France.

Correspondence: Thomas Radtke, University of Zurich, Division of Occupational and Environmental Medicine, Epidemiology, Biostatistics and Prevention Institute, Hirschengraben 84, CH - 8001 Zurich, Switzerland. E-mail: thomas.radtke@uzh.ch

Received: Nov 052018 | Accepted after revision: Jan 082019

Acknowledgements: We kindly thank Julia Braun and Sarah Haile (biostatisticians at the University of Zurich, Zurich, Switzerland) for their support with regard to statistical analyses and data interpretation.

Conflict of interest: None declared.

\section{References}

1 Le Guern V, Mahr A, Mouthon L, et al. Prevalence of systemic sclerosis in a French multi-ethnic county. Rheumatology (Oxford) 2004; 43: 1129-1137.

2 Schoenfeld SR, Castelino FV. Interstitial lung disease in scleroderma. Rheum Dis Clin North Am 2015; 41: 237-248.

3 Lefevre G, Dauchet L, Hachulla E, et al. Survival and prognostic factors in systemic sclerosis-associated pulmonary hypertension: a systematic review and meta-analysis. Arthritis Rheum 2013; 65: 2412-2423.

4 Caron M, Hoa S, Hudson M, et al. Pulmonary function tests as outcomes for systemic sclerosis interstitial lung disease. Eur Respir Rev 2018; 27: 170102.

5 Soumagne T, Guillien A, Chambellan A, et al. Global Lung Initiative equations for pulmonary hypertension screening in systemic sclerosis. Eur Respir J 2018; 52: 1800528.

6 Coghlan JG, Wolf M, Distler O, et al. Incidence of pulmonary hypertension and determining factors in patients with systemic sclerosis. Eur Respir J 2018; 51: 1701197.

7 Sivova N, Launay D, Wemeau-Stervinou L, et al. Relevance of partitioning DLCO to detect pulmonary hypertension in systemic sclerosis. PLoS One 2013; 8: e78001.

8 Degano B, Soumagne T, Delaye T, et al. Combined measurement of carbon monoxide and nitric oxide lung transfer does not improve the identification of pulmonary hypertension in systemic sclerosis. Eur Respir J 2017; 50: 1701008. 
9 Zavorsky GS, Hsia CC, Hughes JM, et al. Standardisation and application of the single-breath determination of nitric oxide uptake in the lung. Eur Respir J 2017; 49: 1600962.

10 van der Lee I, Zanen P, Stigter N, et al. Diffusing capacity for nitric oxide: reference values and dependence on alveolar volume. Respir Med 2007; 101: 1579-1584.

11 Zavorsky GS, Cao J, Murias JM. Reference values of pulmonary diffusing capacity for nitric oxide in an adult population. Nitric Oxide 2008; 18: 70-79.

12 Aguilaniu B, Maitre J, Gienet S, et al. European reference equations for CO and NO lung transfer. Eur Respir J 2008; 31: 1091-1097.

13 Munkholm M, Marott JL, Bjerre-Kristensen L, et al. Reference equations for pulmonary diffusing capacity of carbon monoxide and nitric oxide in adult Caucasians. Eur Respir J 2018; 52: 1500677.

14 Stanojevic S, Graham BL, Cooper BG, et al. Official ERS technical standards: Global Lung Function Initiative reference values for the carbon monoxide transfer factor for Caucasians. Eur Respir J 2017; 50: 1700010.

Copyright @eES 2019 